\title{
Altered left ventricular vortex ring formation by 4-dimensional flow magnetic resonance imaging after repair of atrioventricular septal defects
}

\author{
Emmeline E. Calkoen, MD, ${ }^{a}$ Mohammed S. M. Elbaz, MSc, ${ }^{b}$ Jos J. M. Westenberg, PhD, ${ }^{b}$ \\ Lucia J. M. Kroft, MD, PhD, ${ }^{\mathrm{c}}$ Mark G. Hazekamp, MD, PhD, ${ }^{\mathrm{d}}$ Arno A. W. Roest, MD, PhD, ${ }^{\mathrm{a}}$ and \\ Rob J. van der Geest, $\mathrm{PhD}^{\mathrm{b}}$
}

\begin{abstract}
Objectives: During normal left ventricular (LV) filling, a vortex ring structure is formed distal to the left atrioventricular valve (LAVV). Vortex structures contribute to efficient flow organization. We aimed to investigate whether LAVV abnormality in patients with a corrected atrioventricular septal defect (AVSD) has an impact on vortex ring formation.
\end{abstract}

Methods: Whole-heart 4D flow MRI was performed in 32 patients (age: $26 \pm 12$ years), and 30 healthy subjects (age: $25 \pm 14$ years). Vortex ring cores were detected at peak early (E-peak) and peak late filling (A-peak). When present, the 3-dimensional position and orientation of the vortex ring was defined, and the circularity index was calculated. Through-plane flow over the LAVV, and the vortex formation time (VFT), were quantified to analyze the relationship of vortex flow with the inflow jet.

Results: Absence of a vortex ring during E-peak (healthy subjects $0 \%$, vs patients $19 \% ; P=.015$ ), and A-peak (healthy subjects $10 \%$ vs patients $44 \% ; P=.008$ ) was more frequent in patients. In 4 patients, this was accompanied by a high VFT (5.1-7.8 vs $2.4 \pm 0.6$ in healthy subjects), and in another 2 patients with abnormal valve anatomy. In patients compared with controls, the vortex cores had a moreanterior and apical position, closer to the ventricular wall, with a more-elliptical shape and oblique orientation. The shape of the vortex core closely resembled the valve shape, and its orientation was related to the LV inflow direction.

Conclusions: This study quantitatively shows the influence of abnormal LAVV and LV inflow on 3D vortex ring formation during LV inflow in patients with corrected AVSD, compared with healthy subjects. (J Thorac Cardiovasc Surg $2015 ; 150: 1233-40)$

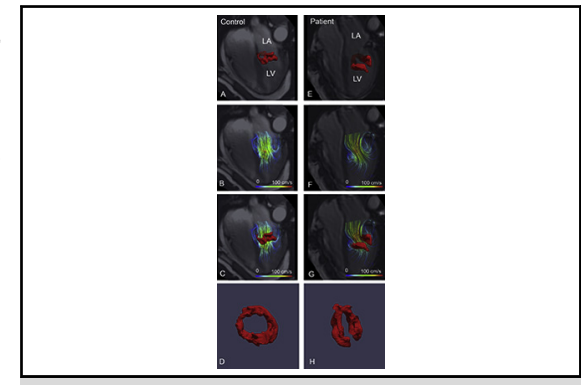

Different vortex core orientation and shape in patient with corrected AVSD compared with control.

Central Message

Atrioventricular valve morphology and abnormal vortex formation in the left ventricle are closely related in patients who have undergone correction for AVSD.

\section{Perspective}

Vortex structures that form in the LV during diastolic filling contribute to efficient flow organization. The 4D flow MRI shows abnormalities of the 3-D vortex cores, which relate to LAVV and inflow abnormalities, in patients who have had AVSD corrected. This study stresses the relationship between LAVV morphology and surgery and abnormal vortex formation

See Editorial Commentary page 1241.

\footnotetext{
From the ${ }^{\mathrm{a}}$ Department of Paediatric Cardiology; ${ }^{\mathrm{b}}$ Department of Radiology, Division of Image Processing; ' Department of Radiology; and ${ }^{\mathrm{d}}$ Department of Thoracic Surgery, Leiden University Medical Center, Leiden, The Netherlands.

E.E. Calkoen is supported by a grant from the Willem-Alexander Kinder-en Jeugdfonds; M.S.M. Elbaz and J.J.M. Westenberg are supported by a grant from the Dutch Technology Foundation (STW), project number 11626.

E.E. Calkoen and M.S.M. Elbaz contributed equally to this work. A.A.W. Roest and R.J. van de Geest contributed equally to this work.

Received for publication May 7, 2015; revisions received July 1, 2015; accepted for publication July 15, 2015; available ahead of print Aug 14, 2015.

Address for reprints: Arno A. W. Roest, MD, PhD, Department of Paediatric Cardiology, Leiden University Medical Center, S-1-P, P.O. Box 9600, 2300 RC Leiden, The Netherlands (E-mail: a.roest@lumc.nl). 0022-5223/\$36.00

Copyright (C) 2015 Published by Elsevier Inc. on behalf of The American Association for Thoracic Surgery

http://dx.doi.org/10.1016/j.jtcvs.2015.07.048
} 


\section{Abbreviations and Acronyms \\ AVSD = atrioventricular septal defect \\ A-peak $=$ peak early filling \\ $\mathrm{EDV}=$ end-diastolic volume \\ E-peak $=$ peak late filling \\ ESV $=$ end-systolic volume \\ LAVV = left atrioventricular valve \\ $\mathrm{LV} \quad=$ left ventricle \\ VFT $=$ vortex formation time \\ $3 \mathrm{D}=3$-dimensional \\ $4 \mathrm{D}$ flow $=4$-dimensional (3-directional and time)}

surgical correction of an AVSD, including closure of the "cleft," may result in restricted opening of the $\mathrm{LAVV}^{4}$ and a more-lateral inflow, ${ }^{5}$ which might affect efficiency of cardiac blood flow in the left ventricle (LV).

Survival after surgical correction is excellent in the current era, but the reoperation rate due to valve regurgitation is high. ${ }^{6,7}$ Long-term follow-up data on cardiac function or exercise capacity after AVSD correction are lacking. However, deterioration of cardiac function and New York Heart Association class is described during pregnancy, when cardiac flow increases. ${ }^{8}$

Recently, the formation of a vortex within the LV during diastole was related to the inflow area through the mitral valve in healthy subjects. ${ }^{9}$ The formation of vortex structures (ie, compact regions of swirling blood flow) in LV blood flow patterns during diastolic filling has recently emerged as a potential novel index for characterizing efficient LV blood flow and evaluating cardiac chamber (dys) function. ${ }^{10}$ During LV filling, a vortex ring structure distal to the mitral valve leaflets and enclosing the inflow jet is observed. This vortical flow is considered an efficient mechanism for transporting a significant portion of LVfilling volume toward the aorta, ${ }^{11}$ minimizing energy loss, and helping mitral valve closure. ${ }^{12,13}$ Recently, 3directional, 3-dimensional (3D) and time-resolved velocity-encoded MRI (magnetic resonance imaging; 4D flow MRI) has been introduced to assess vortex ring formation during LV filling in vivo, ${ }^{9}$ because it has the advantage of a $3 \mathrm{D}$ evaluation of the vortex ring.

Given the relationship between the vortex ring properties and the mitral valve morphology and LV inflow, ${ }^{9,14-17}$ we hypothesized that LAVV abnormalities, and associated abnormal lateral inflow ${ }^{5}$ after surgical AVSD correction, may result in disturbed vortex flow during LV filling. Therefore, we used 4D flow MRI to identify and quantitatively characterize the geometric properties and anatomic location of vortex ring cores during early and late LV filling, allowing quantitative assessment of $3 \mathrm{D}$ vortex ring properties in AVSD-corrected patients and comparison with healthy controls.

\section{METHODS}

\section{Study Population}

The study was approved by the ethical committee of the Leiden University Medical Center, and written informed consent was obtained from all participants or their parents. Thirty-two patients with a history of corrected AVSD were prospectively enrolled from a surgical database. ${ }^{18}$ Thirty healthy subjects of similar age, without a history of cardiac disease, were included for comparison.

Participants in the current study were included in previous studies with the aim of characterizing and quantifying diastolic transatrioventricular flow. ${ }^{5,19}$ Twenty-four of the 30 healthy subjects were included in a study that provided reference values for 3D vortex LV flow. ${ }^{9}$ None of the previously published papers addresses vortex formation in corrected AVSD patients. For clarity, we use the term "left atrioventricular valve" (LAVV) in patients and controls, instead of referring to the mitral valve in healthy subjects, and the LAVV in patients.

\section{Magnetic Resonance Imaging Acquisition and \\ Analysis}

Whole-heart 4D flow was obtained on a 3T MRI scanner (Ingenia; Phillips Medical Systems International, Best, The Netherlands), with a maximal gradient amplitude of $45 \mathrm{mT} / \mathrm{m}$ for each axis, and a slew rate of $200 \mathrm{~T} / \mathrm{m} / \mathrm{s}$, using a combination of FlexCoverage Posterior coil in the table top with a dStream Torso coil, providing up to 32 coil elements for signal reception. Imaging details are reported elsewhere. ${ }^{5}$

In short, a $3 \mathrm{D}$ volume acquisition of the heart was performed with a velocity encoding of $150 \mathrm{~cm} / \mathrm{s}$ in all 3 directions, and spatial resolution of $2.3 \times 2.3 \times 3.0-4.2 \mathrm{~mm}^{3} ; 30$ cardiac phases were retrospectively reconstructed to represent 1 average heartbeat, with a maximal true temporal resolution of $31 \mathrm{~ms}$. Furthermore, to quantify LV volumes and ejection fraction, a left 2-chamber and 4-chamber cine view, and a short-axis cine stack of slices, was acquired with steady-state free-precession sequences as reported elsewhere. ${ }^{5}$ Spatial resolution was $1.0 \times 1.0 \times 8.0 \mathrm{~mm}^{3}$; in addition, for these acquisitions, 30 phases were retrospectively reconstructed.

All acquisitions were performed with free breathing and no respiratory motion control. The cine steady-state free-precession acquisitions were all performed with 3 signal averages to suppress breathing artifacts. After manual segmentation of LV endocardial boundaries, the LV end-diastolic volume (LVEDV), end-systolic volume (ESV), and ejection fraction were calculated. The sphericity index of the LV was calculated as LVEDV $/\left(\pi / 6 \times\right.$ long-axis at end-diastole $\left.{ }^{3}\right)$.

\section{A 3D Vortex Core Analysis Based on the Lambda2 Method}

Using the image analysis workflow described elsewhere, ${ }^{9}$ the cores of vortex structures within the LV blood flow during diastole, as acquired from the 4D flow MRI data, were identified by a single observer using the Lambda2 method. ${ }^{20}$ In short, the Lambda2 method is a fluid-dynamics-based method that uses the gradient properties of the velocity field to identify vortex cores in the flow. For each subject, the vortex cores were identified at the early (E-peak) and late (A-peak) filling, defined from the trans-LAVV flow quantification, described later, and visualized as isosurfaces.

Qualitative visual inspection of the shape of detected vortex cores was performed to determine whether a 3D ring-shaped vortex core was present, defined as a vortex core with a donut-like (torus) shape (Figure 1). If a vortex ring core was detected during E-peak and/or A-peak, its 3D position (in normalized cylindrical coordinates), orientation, and shape were quantitatively characterized, as illustrated in Figure 1.

\section{Trans-Left Atrioventricular Valve Flow}

Trans-LAVV flow was quantified using the 4D flow MRI data and retrospective valve tracking. ${ }^{21}$ From the through-plane LAVV velocity map, a 


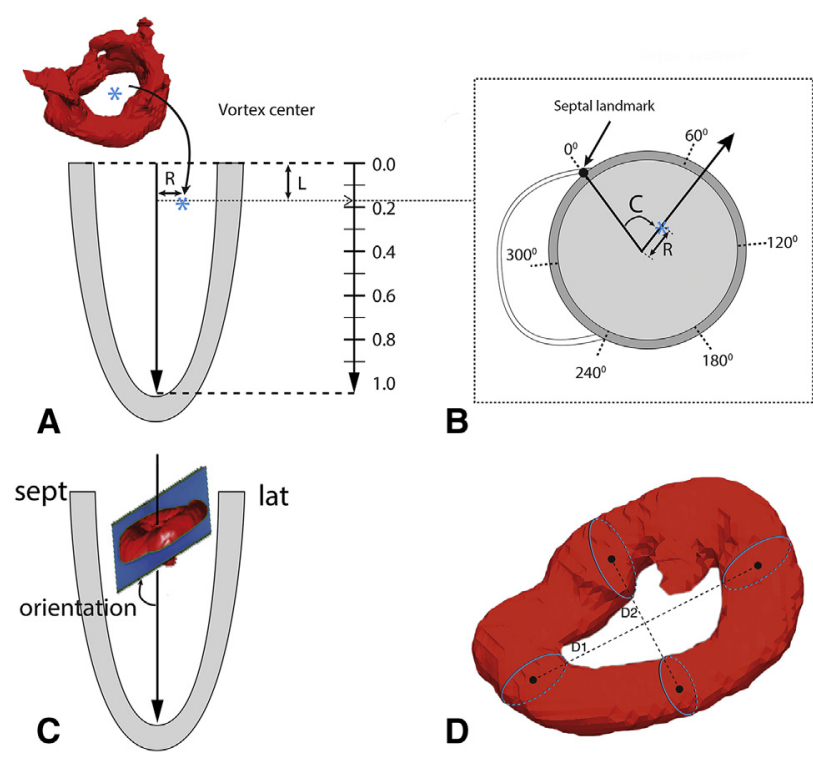

FIGURE 1. The 3-dimensional quantitative vortex core parameters. The cylindrical position of the (A) vortex core center (asterisk) was defined using $\mathrm{L}, \mathrm{C}$, and $\mathrm{R}$ coordinates relative to $\mathrm{LV}(\mathrm{A}, \mathrm{B})$. $\mathrm{L}$ and $\mathrm{R}$ were normalized relative to the LV long-axis length and the radius of the LV endocardial cavity, respectively. The orientation angle was defined relative to the long-axis (C). The circularity index was defined as the ratio between the longest (D1) and shortest (D2) diameter (D). (modified after Elbaz and colleagues ${ }^{9}$ ). $R$, Radial; $L$, longitudinal; $C$, circumferential; sept, septal; lat, lateral; D1, longest diameter; $D 2$, shortest diameter.

flow-time curve of the LAVV flow was computed, and E-peak and A-peak were defined. The early LV filling fraction was calculated as (E-wave inflow volume/total inflow volume) $\times 100 \%$. To study the association with diastolic vortex formation, LAVV and inflow characteristics were evaluated. The inflow area and peak velocity during E-peak were quantified at the level of peak inflow velocity. The peak velocity inflow angle (ie, angle between the long-axis and inflow direction) at E-peak was measured using streamline visualization of the flow velocity field on the 4-chamber view, as previously described.

\section{Vortex Formation Time Index}

The vortex formation time (VFT), a dimensionless index previously proposed to quantify the process of vortex progression during early filling, ${ }^{22}$ was determined using the formula: $\mathrm{VFT}=\left(\mathrm{V}_{\mathrm{avg}} \times \mathrm{E}_{\text {duration }}\right) / \mathrm{D}$, based on the average speed of blood flow during the early filling period $\left(\mathrm{V}_{\text {avg }}\right)$, the duration of the $\mathrm{E}$-filling $\left(\mathrm{E}_{\text {duration }}\right)$, and the maximum diameter (D). The D denominator was computed at E-peak from the area of the LAVV flow, measured on the velocity map after retrospective valve tracking at peak velocity level (ie, tip of the valves). The diameter was calculated as D, assuming the inflow area to be circular.

\section{Statistical Analysis}

Data analysis was performed using SPSS (version 20.0, SPSS, Inc, Chicago, Ill). Variables were tested for normal distribution using the ShapiroWilk test. Continuous variables are expressed as mean $\pm \mathrm{SD}$, or as median with interquartile range (IQR), as appropriate. Differences between presence of E-peak versus A-peak vortex ring core were tested with a Pearson $\chi^{2}$ analysis. Differences between patients and controls, and subjects with versus without E-peak vortex ring core, were assessed using an unpaired $t$ test or a Mann-Whitney $U$ test. Correlation between inflow direction,
LV volume, and vortex position parameters were assessed with linear regression analysis (Pearson r).

\section{RESULTS \\ Patient Characteristics}

Characteristics of patients and healthy controls are presented in Table 1. Of 32 patients, 1 had a double-orifice $\mathrm{LAVV}^{3}, 1$ had a single papillary muscle, ${ }^{3}$ and another was known to have dextrocardia. In patients compared with controls, the mean LV ejection fraction was lower, diastasis was shorter, and the LV sphericity index was higher.

\section{Presence of 3D Vortex Cores During E-Peak and A-Peak}

In all controls, during peak E-filling, a quasi-ring-shaped vortex core was identified distal to the mitral valve in the LV blood flow pattern (Figure 2). In $26(81 \% ; P=.015)$ patients, such a compact $3 \mathrm{D}$ vortex ring core distal to the LAVV was identified during peak E-filling. The shape of the detected 3D vortex ring cores in patients was more frequently deformed, albeit that the vortex cores were still compact and recognizable (Figure 2).

Visually, the shape of the vortex core tended to resemble the shape of the inflow area over the LAVV, as observed on the through-plane velocity maps (Figure 2). In 6 patients $(19 \%)$ (Table 2), no E-peak vortex ring core was detected; instead, only a complex irregular vortex shape was present. The 6 patients included the patient with a double-orifice LAVV (Figure E1), and the patient with a single papillary muscle. The other 4 patients without an E-peak vortex ring core had a small LAVV area, higher peak velocity, and VFT deviating more than $2 \mathrm{SD}$ (VFT $=5.1,5.5,7.4$, and 7.8) (Table 2) from that for healthy participants $(2.6 \pm 0.6)$. The other patients with an E-peak vortex ring core had a mean VFT of $2.4 \pm 0.6$, which was very similar to the healthy participants. The LV shape parameters LVEDV and sphericity index, of the 6 patients without Epeak vortex, still fell within the ranges of the patients with an E-peak vortex ring core.

At peak A-filling, an asymmetric compact vortex ring core formed at the basal LV level in 27 healthy subjects $(90 \%)$, but only $19(59 \% ; P=.006)$ patients. Details of patients with and without A-peak vortex ring core are presented in Table 3. Patients and healthy subjects without an A-peak vortex ring core had shorter diastasis $(14 \pm 17 \mathrm{~ms})$, compared with patients and controls with an A-peak vortex ring $(109 \pm 85 \mathrm{~ms} ; P<.001)$.

\section{Quantitative 3D Parameters of Vortex Ring Cores and Association With the LAVV and LV Characteristics}

The circumferential, longitudinal, and radial position, orientation, and circularity index were quantified for all detected vortex ring cores. During E-peak and A-peak, the 
TABLE 1. Characteristics of healthy subjects and patients

\begin{tabular}{|c|c|c|}
\hline Characteristics & $\begin{array}{l}\text { Healthy } \\
\text { subjects }\end{array}$ & $\begin{array}{c}\text { Patients with } \\
\text { corrected AVSD }\end{array}$ \\
\hline Age $(y)$ & $23(13-38)$ & $26 \pm 12$ \\
\hline Male $(\%)$ & $14(46)$ & $9(28)$ \\
\hline Heart rate $(\mathrm{bpm})$ & $68(60-78)$ & $76 \pm 13$ \\
\hline Diastasis (ms) & $116 \pm 89$ & $26(0-67)^{*}, \dagger$ \\
\hline Type AVSD & - & $\begin{array}{c}21(66 \%) \text { partial } \\
11(33 \%) \text { complete } \\
\text { or intermediate }\end{array}$ \\
\hline Time after surgical correction (y) & & $20 \pm 9$ \\
\hline Stroke volume LV (mL) & $89 \pm 23$ & $85 \pm 19$ \\
\hline LVEDV (mL) & $146 \pm 42$ & $155 \pm 33$ \\
\hline LVEDV/BSA $\left(\mathrm{mL} / \mathrm{m}^{2}\right)$ & $87 \pm 13$ & $91 \pm 15$ \\
\hline LV sphericity index & $0.37 \pm 0.06$ & $0.57 \pm 0.14 \ddagger$ \\
\hline Inflow area $\left(\mathrm{cm}^{2}\right)$ & $9.2 \pm 2.0$ & $8.5 \pm 2.5$ \\
\hline Peak velocity $(\mathrm{cm} / \mathrm{s})$ & $94 \pm 15$ & $93(77-145)$ \\
\hline VFT index & $2.6 \pm 0.6$ & $2.4(1.9-3.1)$ \\
\hline Blood pressure systolic ( $\mathrm{mm} \mathrm{Hg}$ ) & $112 \pm 13$ & $119 \pm 20$ \\
\hline Blood pressure diastolic $(\mathrm{mm} \mathrm{Hg})$ & $67 \pm 9$ & $67 \pm 12$ \\
\hline $\mathrm{E} / \mathrm{A}$ ratio peak flow rate & $2.5 \pm 0.8$ & $2.1(1.7-2.6)^{*}$ \\
\hline Early filling fraction $(\%)$ & $76 \pm 5$ & $73 \pm 13$ \\
\hline Ejection fraction $(\%)$ & $61 \pm 5$ & $56(52-58) \ddagger$ \\
\hline LAVV regurgitation $(\%)$ & - & $14 \pm 8$ \\
\hline
\end{tabular}

Boldface indicated the time after surgical correction. AVSD, Atrioventricular septal defect; $b p m$, beats per minute; $L V$, left ventricle; $L V E D V$, left ventricular enddiastolic volume; $B S A$, body surface area; $V F T$, vortex formation time; $L A V V$, left atrioventricular valve; $E / A$, early/late filling. ${ }^{*}$ Excluding 2 cases without late-peak filling. $\dagger P<.01 \ddagger P<.001$.

center of the vortex ring core was positioned more anteriorly (lower circumferential value), closer to the apex (higher longitudinal value), and closer to the LV wall (higher radial value) in patients, compared with control subjects (Table 4).

In healthy subjects, the vortex orientation ranged from $55^{\circ}$ to $88^{\circ}$, whereas in patients, the vortex orientation showed a wider range $\left(14^{\circ}-134^{\circ}\right)$. Three patients had a vortex orientation angle $>90^{\circ}$ (ie, $102^{\circ}, 115^{\circ}$, and $134^{\circ}$ ), indicating a reversed orientation of the ring (Figure E2), with the lateral side of the vortex being positioned toward the apex, in contrast to the control subjects, for whom the septal side was positioned more apically. In patients with nonreversed vortex ring cores (orientation $<90^{\circ}$ ), cores were in a moretilted position $\left(50^{\circ} \pm 20^{\circ}\right)$, compared with the position in healthy subjects $\left(71^{\circ} \pm 9^{\circ} ; P<.001\right)$ (Figure 3). During A-peak, the vortex ring core orientation was in a nonreversed, more-tilted position in all patients, compared with control subjects $\left(54^{\circ} \pm 21^{\circ}\right.$ vs $\left.72^{\circ} \pm 6^{\circ} ; P=.001\right)$. During both E-peak and A-peak, vortex ring cores were less circular in patients, compared with healthy subjects (Figure 3; Table 4).

In patients, vortex ring core orientation angle relative to the LV long-axis showed a significant correlation with the inflow angle $($ E-peak $\mathrm{r}=0.41 ; P=.037$; A-peak $\mathrm{r}=.62$; $P=.005)$; inflow area during E-peak $(\mathrm{r}=0.47$; $P=.015)$; and LVEDV (E-peak $\mathrm{r}=0.61 ; P=.001$;
A-peak $\mathrm{r}=0.54 ; P=.017)$. In healthy subjects, no significant correlations were found between orientation angle and inflow angle, inflow area, or LVEDV. In patients, the sphericity index of the LV did not show a relationship with vortex ring core characteristics. Ejection fraction did not correlate with vortex ring core presence or characteristics. In patients, no significant correlation $(P=.97)$ was found between regurgitation fraction and vortex orientation.

\section{DISCUSSION}

This study, for the first time, quantitatively describes the effect of LAVV abnormalities and abnormal LV inflow on $3 \mathrm{D}$ vortex ring formation in LV blood flow patterns during early and late filling, in patients with surgically corrected LAVV valves compared with healthy subjects. These findings highlight the close relationship between AVV morphology and LV filling characteristics, with LV vortex formation. Previous studies on vortex formation in the presence of LAVV abnormalities were performed in vitro, or were based on 2-dimensional analysis using echocardiography.

The current report provides an in vivo 3D analysis using 4D flow MRI. Key findings are as follows: (1) Absence of vortex ring formation is more frequent in corrected-AVSD patients and is related to LAVV abnormalities (single papillary muscle and double-orifice) and a narrow LAVV diameter concomitant with high inflow velocities evidenced by a high VFT. (2) If a vortex ring core is present in correctedAVSD patients, it has a different position, a moreelliptical shape, and an oblique orientation compared with controls, and these differences correlate with LV inflow direction.

\section{Absence of Vortex Ring Core Related to Valve Morphology and VFT}

In the normal heart, adequate suction, correct shape of the valve leaflets, and normal electrical conduction allow vortex ring formation during LV filling. ${ }^{23}$ In our study, 6 patients did not develop a vortex ring. One of these patients had a double-orifice LAVV, resulting in 2 inflow jets, as is seen after edge-to-edge repair. Absence of a ring in this patient is in agreement with computational fluid dynamics studies simulating edge-to-edge repair, resulting in abnormal vortex formation with increased energy loss and decreased LV filling efficiency. ${ }^{24}$ Another patient without vortex ring formation had a single papillary muscle, stressing the contribution of the papillary muscles to the shape of the vortex ring. ${ }^{25}$

Both cases underline the influence of morphologic LAVV abnormalities on vortex formation. Next to 2 patients with abnormal anatomy, 4 other patients were observed without E-peak vortex ring formation. These 4 had a VFT more than 2 SDs higher, compared with patients with an E-peak vortex 


\section{Control}

E-peak
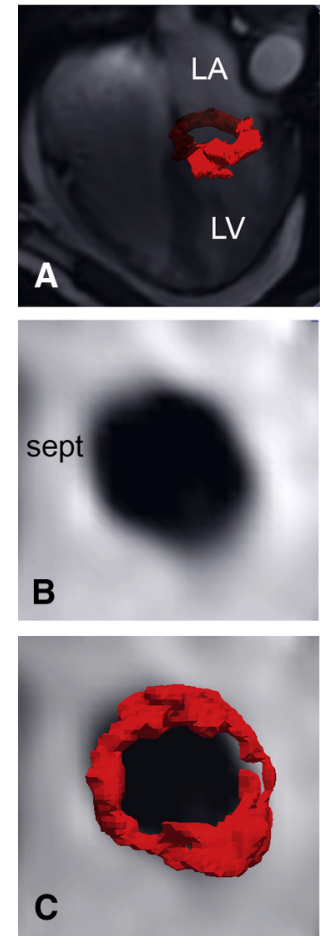

A-peak
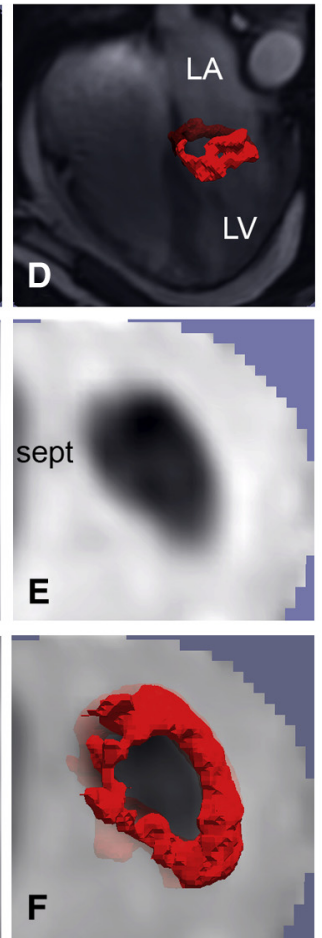

Corrected AVSD patient

E-peak
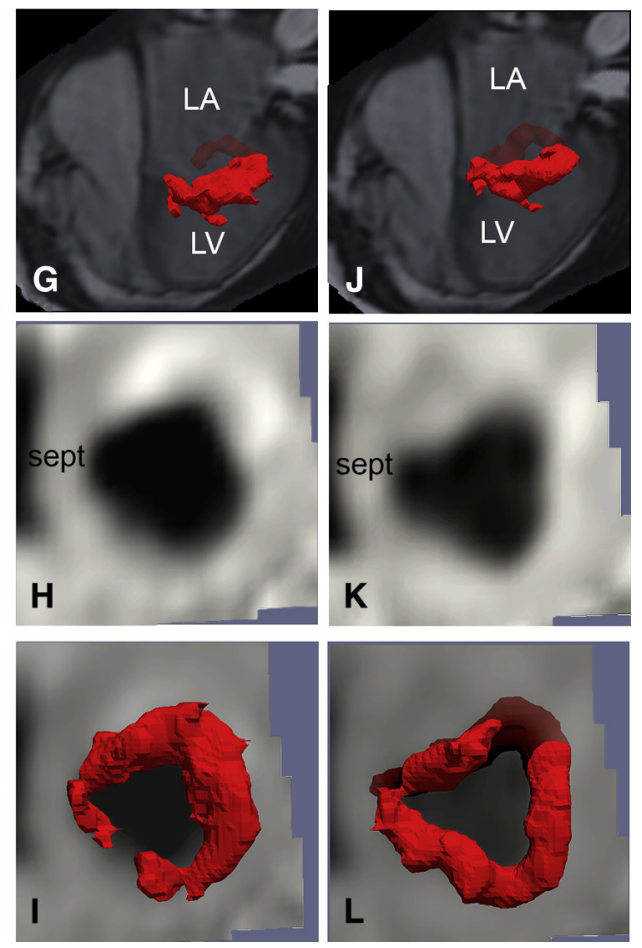

FIGURE 2. Shape of vortex core corresponds with the inflow area. Vortex cores depicted on reformat planes of through-plane flow at peak inflow velocity $(\mathrm{B}, \mathrm{E}, \mathrm{H}, \mathrm{K})$ during E-peak and A-peak in a healthy subject (A-F) and a patient (G-L). AVSD, Atrioventricular septal defect; E-peak, early filling; A-peak, late filling; $L A$, left atrium; $L V$, left ventricle; Sept, septal side.

ring core and with healthy subjects. The VFT index, studied in vitro and in vivo, is known to have an optimal value range that defines efficient vortex formation. ${ }^{22,26}$ In patients with mitral stenosis, higher values of VFT have been related to the absence of a well-formed vortex ring, resulting in increased energy dissipation. ${ }^{27}$

In the current MRI study, the VFT values in healthy subjects were lower than the reported range (3.5-5.5) measured with echocardiography. ${ }^{22}$ This difference might be due to differences between modalities ${ }^{28}$ and their definition of valve diameter measurement. However, the markedly higher VFT values in patients with an absent vortex ring confirms that patients with a narrow LAVV diameter and a higher peak velocity develop abnormal vortex flow. ${ }^{22,26}$ In addition, VFT measurements were comparable and not significantly different between controls and patients when an E-vortex ring core was present. Absence of a separate A-peak vortex ring core was related to the shorter diastasis in patients, similar to what is reported in healthy controls.

TABLE 2. Characteristics of the 6 patients without a vortex ring core during E-peak or A-peak

\begin{tabular}{lcccccc}
\hline \multicolumn{1}{c}{ Characteristics } & Pt 1 & Pt 2 & Pt 3 & Pt 4 & Pt 5 & Pt 6 \\
\hline Age (y) & 10 & 23 & 20 & 36 & 12 & 32 \\
Type AVSD & Partial double-orifices & $\begin{array}{c}\text { Complete single papillary } \\
\text { muscle }\end{array}$ & Partial & Complete & Complete & Complete dextrocardia \\
Regurgitation (\%) & 10 & 23 & 20 & 36 & 12 & \\
Heart rate (bpm) & 76 & 78 & 96 & 114 & 60 & 32 \\
Diastasis (ms) & 24 & 0 & No A-peak & No A-peak & 0 & 62 \\
Early filling fraction (\%) & 61 & 80 & 100 & 100 & 81 & 0 \\
Area LAVV $\left(\mathrm{cm}^{2}\right)$ & 9.2 & 8.4 & 6.0 & 5.1 & 4.6 & 88 \\
Peak velocity $(\mathrm{cm} / \mathrm{s})$ & 58 & 110 & 144 & 158 & 146 & 4.6 \\
VFT & 1.6 & 2.5 & 5.1 & 5.5 & 7.8 & 155 \\
\hline
\end{tabular}

AVSD, Atrioventricular septal defect; $P t$, patient; A-peak, late-peak filling; $b p m$, beats per minute; $L A V V$, left atrioventricular valve; $V F T$, vortex formation time. 
TABLE 3. Characteristics of healthy subjects and patients with a vortex ring core present during E-peak

\begin{tabular}{|c|c|c|c|c|}
\hline Characteristics & $\begin{array}{c}\text { Healthy subjects; E- and } \\
\text { A-peak ring } \\
\end{array}$ & $\begin{array}{c}\text { Healthy subjects; only } \\
\text { E-peak ring }\end{array}$ & $\begin{array}{c}\text { Patients, E- and } \\
\text { A-peak ring } \\
\end{array}$ & $\begin{array}{c}\text { Patients, only } \\
\text { E-peak ring } \\
\end{array}$ \\
\hline $\mathrm{n}$ & 27 & 3 & 19 & 7 \\
\hline Age (y) & $26 \pm 13$ & $11 \pm 2$ & $26 \pm 14$ & $28 \pm 8$ \\
\hline Type AVSD & - & - & $\begin{array}{l}14 \text { partial } \\
5 \text { complete }\end{array}$ & $\begin{array}{c}5 \text { partial } \\
2 \text { complete }\end{array}$ \\
\hline Regurgitation (\%) & - & - & $12 \pm 8$ & $13 \pm 6$ \\
\hline Heart rate (bpm) & $67 \pm 10$ & $95 \pm 7$ & $72 \pm 12$ & $80 \pm 4$ \\
\hline Diastasis (ms) & $128 \pm 86$ & $7 \pm 13$ & $81 \pm 79$ & $22 \pm 18$ \\
\hline Passive filling fraction (\%) & $76 \pm 5$ & $79 \pm 2$ & $72 \pm 11$ & $68 \pm 10$ \\
\hline Area LAVV $\left(\mathrm{cm}^{2}\right)$ & $9.5 \pm 1.9$ & $6.4 \pm 0.8$ & $8.8 \pm 2.5$ & $9.3 \pm 2.3$ \\
\hline Peak velocity $(\mathrm{cm} / \mathrm{s})$ & $93 \pm 16$ & $105 \pm 4$ & $92 \pm 15$ & $94 \pm 28$ \\
\hline VFT & $2.6 \pm 0.6$ & $2.6 \pm 0.2$ & $2.4 \pm 0.6$ & $2.4 \pm 0.5$ \\
\hline
\end{tabular}

Values are mean $\pm \mathrm{SD}$, unless otherwise indicated. E-peak, Early-peak filling; A-peak, late-peak filling; $A V S D$, atrioventricular septal defect; $b p m$, beats per minute; LAVV, left atrioventricular valve; VFT, vortex formation time.

\section{Vortex Ring Formation Related to LAVV and LV Characteristics}

In normal hearts, a vortex ring forms at the tip of the $\mathrm{LAVV}^{29}$ with the septal side positioned toward the apex, owing to the unbalanced shape of the leaflets (ie, longer anterior and shorter posterior leaflet) and interaction with the LV wall. ${ }^{23,29}$ The visual similarity between the 3D shape of the vortex ring core and the (abnormal) LAVV orifices (ie, LAVV inflow area) in this study illustrates the impact of the abnormal LAVV on vortex ring formation. Moreover, the observed correlation between disturbed vortex characteristics and altered inflow area and direction, indicates an influence of abnormal valve and inflow on vortex ring formation.

TABLE 4. Quantitative vortex core characteristics, as presented in Figure 1, at E-peak and A-peak, in healthy subjects and patients

\begin{tabular}{lccc}
\hline Characteristics & $\begin{array}{c}\text { Healthy subjects } \\
(\mathbf{n}=\mathbf{3 0})\end{array}$ & $\begin{array}{c}\text { Patients } \\
(\mathbf{n}=\mathbf{2 6})\end{array}$ & $\boldsymbol{P}$ value \\
\hline E-peak & & & \\
$\quad$ Circumferential & $90^{\circ} \pm 26^{\circ}$ & $70^{\circ} \pm 21^{\circ}$ & .003 \\
Longitudinal & $0.19 \pm 0.04$ & $0.23 \pm 0.07$ & .015 \\
Radial & $0.26 \pm 0.07$ & $0.33 \pm 0.08$ & .001 \\
Orientation & $71^{\circ} \pm 9^{\circ}$ & $50^{\circ} \pm 20^{\circ} *$ & $<.001^{*}$ \\
Circularity index & $0.80 \pm 0.08$ & $0.70 \pm 0.13$ & .002 \\
\hline \multicolumn{4}{c}{ Patients } \\
& Healthy Subjects & $(\mathbf{n}=\mathbf{1 9})$ & \\
\hline A-peak & $(\mathbf{n}=\mathbf{2 7})$ & & \\
Circumferential & $106^{\circ} \pm 27^{\circ}$ & $80^{\circ} \pm 28^{\circ}$ & .003 \\
Longitudinal & $0.15 \pm 0.05$ & $0.19 \pm 0.05$ & .004 \\
Radial & $0.20 \pm 0.08$ & $0.32 \pm 0.14$ & .002 \\
Orientation & $72^{\circ} \pm 6^{\circ}$ & $54^{\circ} \pm 21^{\circ}$ & .001 \\
Circularity index & $0.63(0.59-0.69)$ & $0.60 \pm 0.10$ & .115 \\
\hline
\end{tabular}

E-peak, Early-peak filling; A-peak, late-peak filling. *Including patients with a nonreversed orientation $(n=23)$. Inclusion of all patients $(n=26)$ gives a mean orientation of $58 \pm 29 ; P=.037$.
Our findings are in agreement with computational fluid dynamics experiments, ${ }^{16}$ 2-dimensional echocardiography analysis in human, ${ }^{17,30}$ and an MRI study in sheep ${ }^{31}$ showing that LAVV repair and replacement are related to unnatural vortex formation. Aside from the impact of the valve abnormalities, the correlation between vortex ring orientation and LVEDV shows an impact of LV size on vortex formation. This finding is in agreement with the relationship between LVEDV and vortex size observed in patients with dilated cardiomyopathy. ${ }^{32}$

In the current study, no relationship was found between vortex ring core characteristics and sphericity index, as described in a previous study using vortex filling fraction. ${ }^{11}$ However, absence of such a correlation might be due to the narrow range in sphericity index of patients in the current study. In addition, no relationship was found with the ejection fraction, which was close to normal in all patients. Consequently, a possible impact of a restrictive LV remains to be investigated.

\section{Clinical Implications}

During AVSD correction, the common atrioventricular valve is separated and the cleft is closed. During surgical correction of an AVSD, surgeons have to minimize valve regurgitation, without causing valve restriction. Even though the shape of the vortex will not be the main concern of the surgeon during correction of an AVSD, awareness of the effect of valve surgery on the formation of vortices in the LV blood flow is important, as changes in vortex formation might affect blood flow efficiency. Similar consideration accounts for LAVV surgery in other congenital and acquired heart disease.

These results do not preclude the possibility that the aberrant vortex formation is a coping mechanism of the heart and has a favorable effect on cardiac function. However, computational fluid dynamics studies have shown increased energy dissipation in cases with a disturbed LAVV shape 

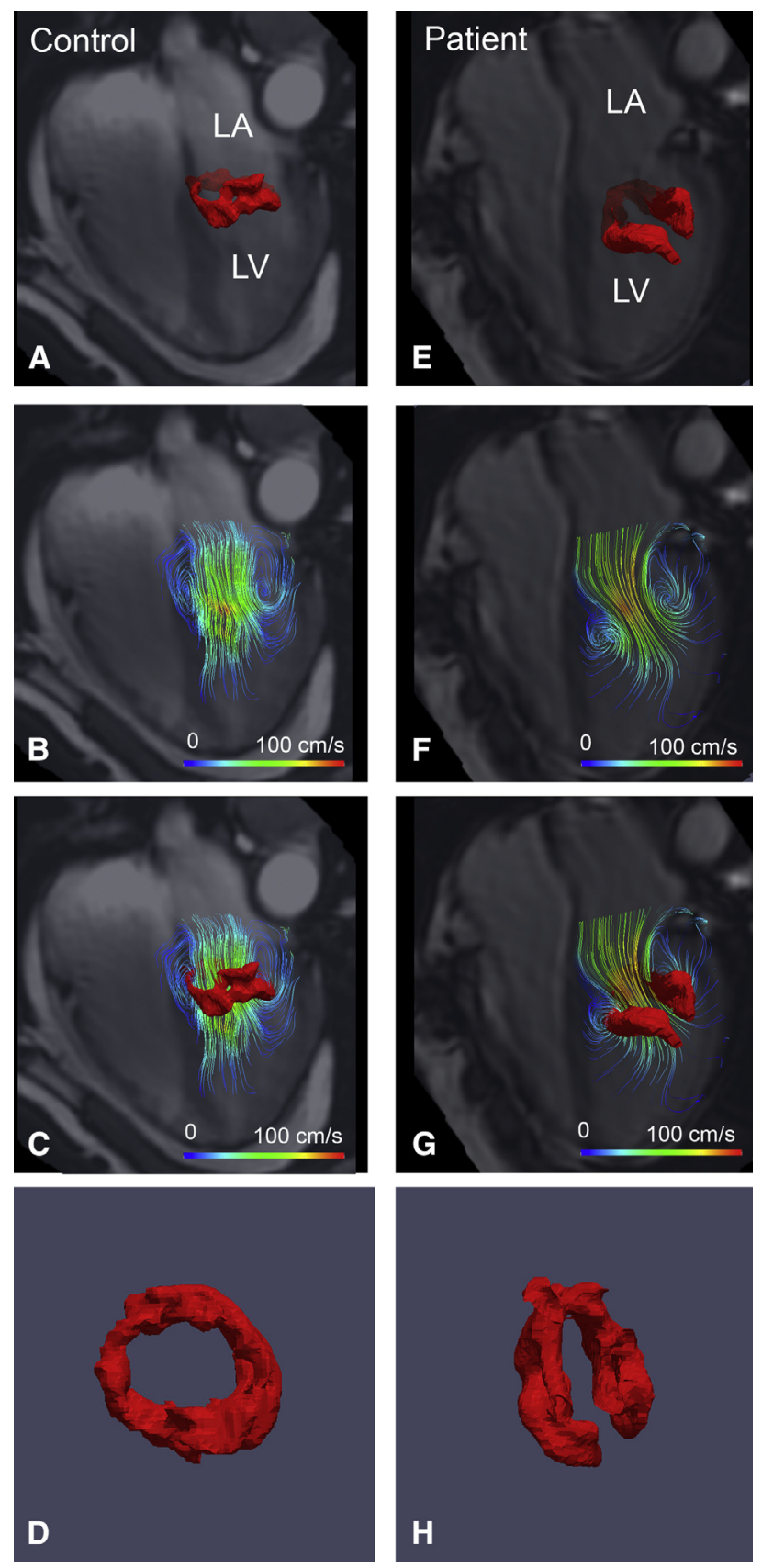

FIGURE 3. Example of a vortex core during early filling in a patient (E-H) and a healthy subject (A-D). Streamline visualization (color coding represents velocity magnitude) shows a more lateral inflow direction in the patient (F) compared with the control (B). The vortex core has a more-tilted orientation $(\mathrm{G})$ and elliptical shape $(\mathrm{H})$ in the patient, compared with the healthy control (C-D). $L A$, Left atrium; $L V$, left ventricle.

and abnormal vortex flow formation, ${ }^{14-16}$ and reduced efficiency of the heart pump in patients with a higher VFT index. ${ }^{33} \mathrm{~A}$ reversed vortex resulted in an increased energy-dissipation level compared with a normally oriented vortex. ${ }^{15}$ Whether the aberrant vortex formation additionally results in clinically relevant changes in flow efficiency in corrected AVSD patients remains to be investigated during long term follow-up of this patient group.

In the current study, global diastolic and systolic functions were within normal ranges; however, vortex ring characteristics were significantly different in patients, compared with healthy subjects. This finding may suggest that vortex formation provides a more sensitive indication of disturbed diastolic function than do conventional functional parameters, and confirms the suggested role of vortex analysis as an early predictor of diastolic dysfunction. ${ }^{34}$ In patients with unbalanced AVSD, the inflow direction may play a role in LV growth as well. ${ }^{35}$ Analysis of vortex formation in unbalanced AVSDs potentially can contribute to predicting LV growth and decision making for biventricular repair.

The disturbed vortex formation observed at rest may become more pronounced during exercise or pregnancy, when cardiac blood flow increases. Future studies are needed to analyze flow organization in situations with increased cardiac flow, such as during exercise. Furthermore, disturbed vortex ring formation might influence shear stress, which serves as an epigenetic factor in cardiac remodeling. ${ }^{13}$ Therefore, future long-term studies have to reveal if abnormal vortex formation affects energy loss or cardiac pumping efficiency, and/or may serve as an early predictor for poor cardiac outcome. ${ }^{30}$

\section{Limitations}

Absence or presence of a vortex ring was scored visually; however, a previous study ${ }^{9}$ showed that vortex detection can be done with low inter- and intra-observer variation. In the current study, the absence of vortex formation was further confirmed by the significant high VFT, which is in line with previous work. The 4D flow MRI has the disadvantage of having a relatively long scan duration (8-10 minutes), and it is associated with relatively high costs, compared with echocardiography.

Vortex core analysis was limited to the E-peak and A-peak; thus, no data were available on the timing, forming, and disappearance of the vortex cores. Parameters of diastolic and systolic function of patients were all close to normal reference values, which made correlation with clinical parameters difficult. The inflow angle of the LV was measured on a 2-dimensional plane, but was compared with a 3D-determined vortex orientation. If a vortex ring core was not elliptical, the circularity index was computed by approximating an ellipse around the deformed vortex shape. This approximation may not fully capture or characterize the deformed vortex ring shape.

\section{CONCLUSIONS}

Quantitative 3D vortex analysis of early- and late-filling vortex ring formation revealed a disturbed vortex ring formation in patients after correction of an AVSD. This disturbance is characterized by either the absence of a formed vortex ring 
or alterations in the geometric properties and location of the formed rings. These findings were associated with abnormal LV inflow and morphology of the LAVV in the studied patients. Using 3D analysis, the current in vivo study quantitatively confirms the relationship between LAVV abnormalities and altered vortex ring formation in the LV.

Our findings highlight the close relationship among AVV abnormalities. In addition, they create awareness of the influence of AVV abnormalities and AVV surgery on LV vortex formation. The exact implications of abnormal vortex formation and possible increased energy loss and cardiac remodeling, owing to aberrant vortex formation, need further investigation.

\section{Conflict of Interest Statement}

E.E. Calkoen is financially supported by a grant from the Willem-Alexander Kinder- en Jeugdfonds. M.S.M. Elbaz and J.J.M. Westenberg are financially supported by a grant from the Dutch Technology Foundation (STW), project number 11626. All other authors have nothing to disclose with regard to commercial support.

\section{References}

1. Penkoske PA, Neches WH, Anderson RH, Zuberbuhler JR. Further observations on the morphology of atrioventricular septal defects. J Thorac Cardiovasc Surg. 1985;90:611-22.

2. Takahashi K, Mackie AS, Thompson R, Al-Naami G, Inage A, Rebeyka IM, et al. Quantitative real-time three-dimensional echocardiography provides new insight into the mechanisms of mitral valve regurgitation post-repair of atrioventricular septal defect. J Am Soc Echocardiogr. 2012;25:1231-44.

3. Draulans-Noe HA, Wenink AC, Quaegebeur J. Single papillary muscle ("parachute valve") and double-orifice left ventricle in atrioventricular septal defect convergence of chordal attachment: surgical anatomy and results of surgery. Pediatr Cardiol. 1990;11:29-35.

4. Ando M, Takahashi Y. Variations of atrioventricular septal defects predisposing to regurgitation and stenosis. Ann Thorac Surg. 2010;90:614-21.

5. Calkoen EE, Roest AA, Kroft LJ, van der Geest RJ, Jongbloed MR, van den Boogaard PJ, et al. Characterization and improved quantification of left ventricular inflow using streamline visualization with 4DFlow MRI in healthy controls and patients after atrioventricular septal defect correction. J Magn Reson Imaging. 2015;41:1512-20.

6. Ginde S, Lam J, Hill GD, Cohen S, Woods RK, Mitchell ME, et al. Long-term outcomes after surgical repair of complete atrioventricular septal defect. J Thorac Cardiovasc Surg. 2015;150:369-74.

7. St Louis JD, Jodhka U, Jacobs JP, He X, Hill KD, Pasquali SK, et al. Contemporary outcomes of complete atrioventricular septal defect repair: analysis of the Society of Thoracic Surgeons Congenital Heart Surgery Database. J Thorac Cardiovasc Surg. 2014;148:2526-31.

8. Drenthen W, Pieper PG, van der Tuuk K, Roos-Hesselink JW, Voors AA, Mostert B, et al. Cardiac complications relating to pregnancy and recurrence of disease in the offspring of women with atrioventricular septal defects. Eur Heart J. 2005;26:2581-7.

9. Elbaz MS, Calkoen EE, Westenberg JJ, Lelieveldt BP, Roest AA, van der Geest RJ. Vortex flow during early and late left ventricular filling in normal subjects: quantitative characterization using retrospectively-gated 4D flow cardiovascular magnetic resonance and three-dimensional vortex core analysis. J Cardiovasc Magn Reson. 2014;16:78.

10. Hong GR, Pedrizzetti G, Tonti G, Li P, Wei Z, Kim JK, et al. Characterization and quantification of vortex flow in the human left ventricle by contrast echocardiography using vector particle image velocimetry. JACC Cardiovasc Imaging. 2008; 1:705-17.

11. Martinez-Legazpi P, Bermejo J, Benito Y, Yotti R, Perez Del Villar C, GonzalezMansilla A, et al. Contribution of the diastolic vortex ring to left ventricular filling. J Am Coll Cardiol. 2014;64:1711-21.
12. Bellhouse BJ. Fluid mechanics of a model mitral valve and left ventricle. Cardiovasc Res. 1972;6:199-210.

13. Pasipoularides A. Diastolic filling vortex forces and cardiac adaptations: probing the epigenetic nexus. Hellenic J Cardiol. 2012;53:458-69.

14. Hu Y, Shi L, Parameswaran S, Smirnov S, He Z. Left ventricular vortex under mitral valve edge-to-edge repair. Cardiovasc Eng Technol. 2010;1:235-43.

15. Pedrizzetti G, Domenichini F, Tonti G. On the left ventricular vortex reversal after mitral valve replacement. Ann Biomed Eng. 2010;38:769-73.

16. Kheradvar A, Falahatpisheh A. The effects of dynamic saddle annulus and leaflet length on transmitral flow pattern and leaflet stress of a bileaflet bioprosthetic mitral valve. J Heart Valve Dis. 2012;21:225-33.

17. Faludi R, Szulik M, D'hooge J, Herijgers P, Rademakers F, Pedrizzetti G, et al. Left ventricular flow patterns in healthy subjects and patients with prosthetic mitral valves: an in vivo study using echocardiographic particle image velocimetry. J Thorac Cardiovasc Surg. 2010;139:1501-10.

18. Hoohenkerk GJ, Bruggemans EF, Rijlaarsdam M, Schoof PH, Koolbergen DR, Hazekamp MG. More than 30 years' experience with surgical correction of atrioventricular septal defects. Ann Thorac Surg. 2010;90:1554-61.

19. Calkoen EE, Westenberg JJ, Kroft LJ, Blom NA, Hazekamp MG, Rijlaarsdam ME, et al. Characterization and quantification of dynamic eccentric regurgitation of the left atrioventricular valve after atrioventricular septal defect correction with 4D Flow cardiovascular magnetic resonance and retrospective valve tracking. J Cardiovasc Magn Reson. 2015;17:18.

20. Jeong J, Hussain F. On the identification of a vortex. J Fluid Mech. 1995;285:69-94.

21. Westenberg JJ, Roes SD, Ajmone MN, Binnendijk NM, Doornbos J, Bax JJ, et al. Mitral valve and tricuspid valve blood flow: accurate quantification with 3D velocity-encoded MR imaging with retrospective valve tracking. Radiology. 2008;249:792-800.

22. Gharib M, Rambod E, Kheradvar A, Sahn DJ, Dabiri JO, Gharib M, et al. Optimal vortex formation as an index of cardiac health. Proc Natl Acad Sci US A. 2006;103:6305-8.

23. Kheradvar A, Assadi R, Falahatpisheh A, Sengupta PP. Assessment of transmitral vortex formation in patients with diastolic dysfunction. J Am Soc Echocardiogr. 2012;25:220-7.

24. Du D, Jiang S, Wang Z, Hu Y, He Z. Effects of suture position on left ventricular fluid mechanics under mitral valve edge-to-edge repair. Biomed Mater Eng. 2014;24:155-61.

25. Toger J, Kanski M, Carlsson M, Kovacs SJ, Soderlind G, Arheden H, et al. Vortex ring formation in the left ventricle of the heart: analysis by 4D flow MRI and Lagrangian coherent structures. Ann Biomed Eng. 2012;40:2652-62.

26. Dabiri JO, Gharib M. The role of optimal vortex formation in biological fluid transport. Proc Biol Sci. 2005;272:1557-60.

27. Kheradvar A, Pedrizzetti G. Vortex formation in the heart. Vortex formation in the cardiovascular system. London: Springer; 2012. 70-2.

28. Paelinck BP, de Roos A, Bax JJ, Bosmans JM, van der Geest RJ, Dhondt D, et al. Feasibility of tissue magnetic resonance imaging: a pilot study in comparison with tissue Doppler imaging and invasive measurement. J Am Coll Cardiol. 2005;45:1109-16.

29. Kilner PJ, Yang GZ, Wilkes AJ, Mohiaddin RH, Firmin DN, Yacoub MH. Asymmetric redirection of flow through the heart. Nature. 2000;404:759-61.

30. Sengupta PP, Narula J, Chandrashekhar Y. The dynamic vortex of a beating heart: wring out the old and ring in the new! J Am Coll Cardiol. 2014;64:1722-4.

31. Machler H, Reiter G, Perthel M, Reiter U, Bergmann P, Zink M, et al. Influence of a tilting prosthetic mitral valve orientation on the left ventricular flow-an experimental in vivo magnetic resonance imaging study. Eur J Cardiothorac Surg. 2007;32:102-7.

32. Bermejo J, Benito Y, Alhama M, Yotti R, Martinez-Legazpi P, Del Villar CP, et al. Intraventricular vortex properties in nonischemic dilated cardiomyopathy. Am J Physiol Heart Circ Physiol. 2014;306:H718-29.

33. Jiamsripong P, Calleja AM, Alharthi MS, Dzsinich M, McMahon EM, Heys JJ, et al. Impact of acute moderate elevation in left ventricular afterload on diastolic transmitral flow efficiency: analysis by vortex formation time. J Am Soc Echocardiogr. 2009;22:427-31.

34. Pedrizzetti G, La CG, Alfieri O, Tonti G. The vortex-an early predictor of cardiovascular outcome? Nat Rev Cardiol. 2014;11:545-53.

35. Overman DM, Dummer KB, Moga FX, Gremmels DB. Unbalanced atrioventricular septal defect: defining the limits of biventricular repair. Semin Thorac Car diovasc Surg Pediatr Card Surg Annu. 2013;16:32-6.

Key Words: atrioventricular septal defect, atrioventricular valves, vortex flow, 4D flow MRI 

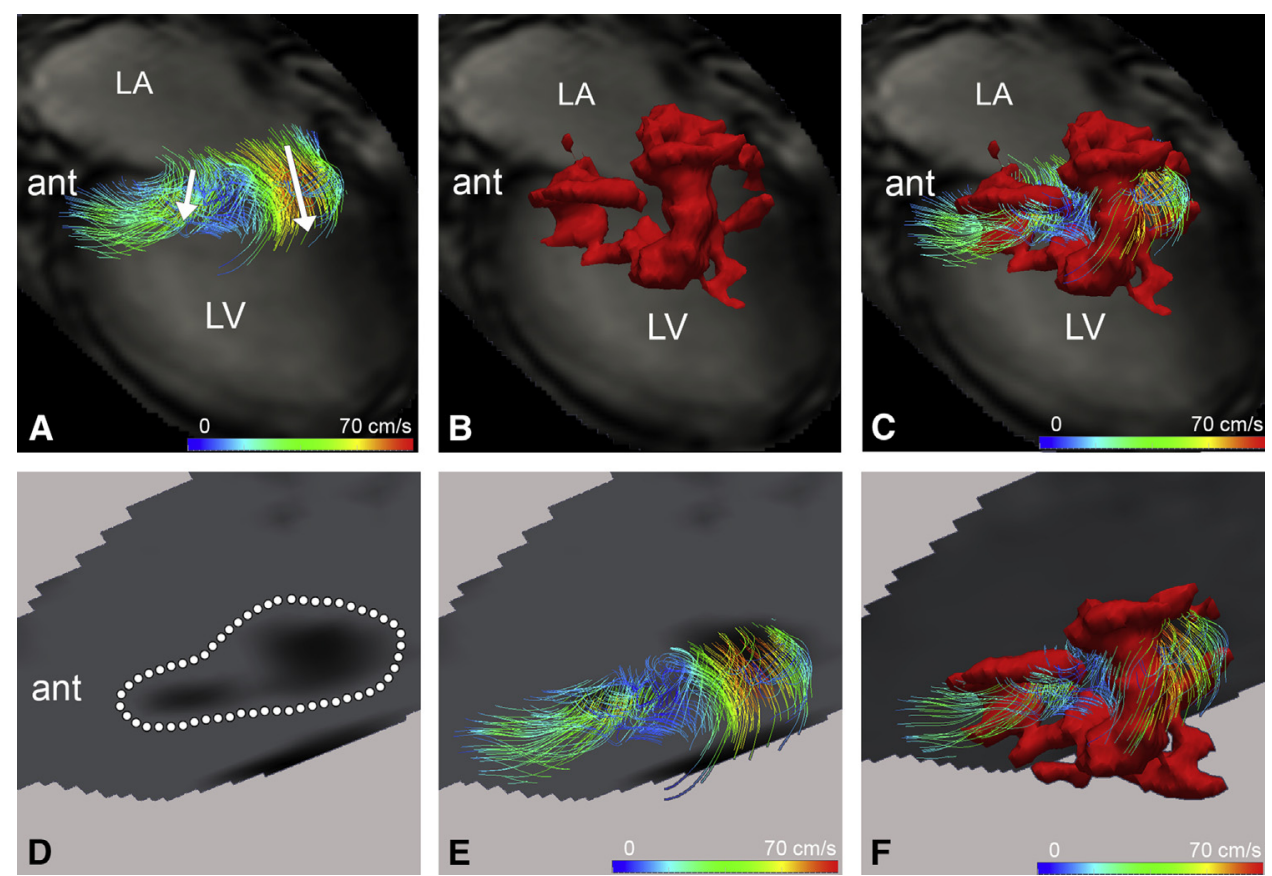

FIGURE E1. Patient with a double-orifice LAVV showed 2 separate inflow jets. Streamlines (color coding representing velocity magnitude) show (A) 2 inflow jets (white arrows). The vortex core had a complex shape (B), but fit with streamlines (C). (D) Through-plane flow analysis showed3 jets in the LAVV (dotted line). Positioning the streamlines (E) and vortex core (F) on top of the velocity map shows that a core is formed around both jets. $L A$, Left atrium; $L V$, left ventricle.
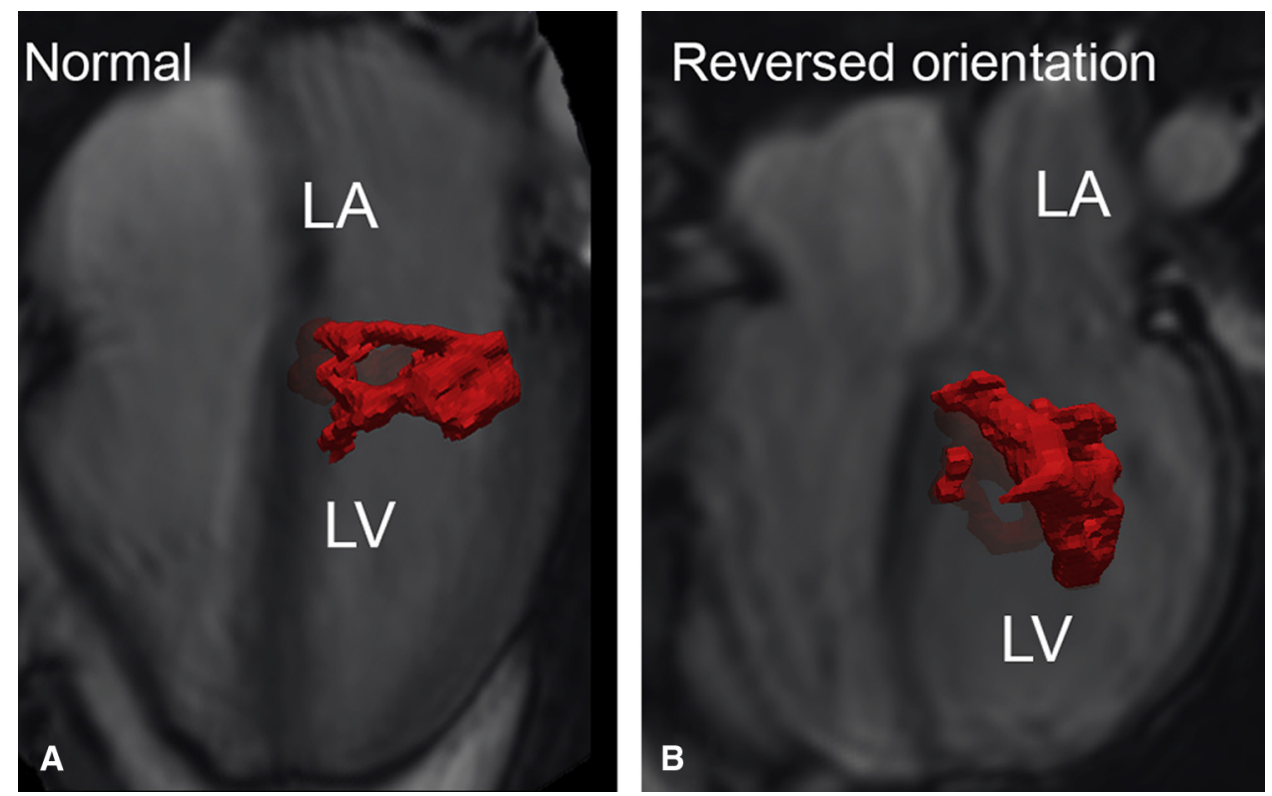

FIGURE E2. Reversed orientation of the vortex ring core in a corrected AVSD patient. Healthy subject (A), and patients (B). $L A$, Left atrium; $L V$, left ventricle; ant, anterior. 